\title{
Transesophageal Echocardiography-Guided WATCHMAN Implantation Without Contrast Use: A Three-Year, Single-Center Experience
}

\author{
Muhammad Hamza Saad Shaukat ${ }^{1}$, Hiren Patel ${ }^{2}$, Rizwan Alimohammad ${ }^{3}$, Augustin DeLago ${ }^{3}$ \\ 1. Internal Medicine, Albany Medical College, Albany, USA 2. Cardiology, Albany Medical College, Albany, USA 3. \\ Cardiology, Capital Cardiology Associates, Albany Medical Center, Albany, USA
}

Corresponding author: Muhammad Hamza Saad Shaukat, hamzasaad1991@gmail.com

\section{Abstract \\ Background}

It is unclear if the WATCHMAN device (Boston Scientific, St. Paul, Minnesota) can be implanted without contrast to prevent complications in patients with advanced chronic kidney disease (CKD) or contrast allergy.

\section{Objective}

The efficiency and safety of WATCHMAN implantation under transesophageal echocardiography (TEE)guidance and fluoroscopy without contrast use.

\section{Methods}

This was a retrospective single-center study at Albany Medical Center between June 2016 and June 2019. Consecutive procedure notes for all WATCHMAN devices implanted between June 2016 and June 2019 were screened to identify patients who did not receive contrast. Patients with incomplete information on the calculation of the 'Congestive heart failure, Hypertension, Age [>75 years], Stroke, Diabetes mellitus, Vascular disease, Age [65 to 74 years], Sex category' (CHA2DS2VASc)/'Hypertension, Abnormal renal/liver function, Stroke, Bleeding history, Labile international normalized ratio, Elderly, Drugs/alcohol' (HASBLED) score and reason(s) precluding safe contrast use were excluded. Efficiency was measured as i) accuracy of device size estimation based on TEE-measured left atrial appendage (LAA) dimensions were determined by the need to change the size of the device initially selected, ii) number of implantation attempts, irrespective of change in device size, iii) whether more than one device was used secondary to inaccurate initial size estimation or other procedural complexities, and iv) successful LAA seal on TEE immediately and 45 -days post-implantation (peri-device leak of $<5 \mathrm{~mm}$ by color Doppler). Procedurerelated complications, immediate and delayed (0-45 days), were recorded.

Received 05/02/2020

Review began 05/21/2020 Review ended 05/22/2020 Published 05/31/2020

(c) Copyright 2020 Saad Shaukat et al. This is an open access article distributed under the terms of the Creative Commons Attribution License CC-BY 4.0., which permits unrestricted use, distribution, and reproduction in any medium, provided the original author and source are credited.

\section{Results}

Twelve patients received WATCHMAN without contrast. The mean age was 79.2 years, with male predominance $(n=8)$. The mean CHA2DS2VASc and HAS-BLED scores were $5.50(+/-1.24)$ and $4.08(+/-1.08)$, respectively. Contrast was avoided because of a history of CKD stage IV $(n=5)$, rapidly progressive CKD stage III $(n=1)$, and contrast allergy $(n=6)$. In 11 out of 12 patients, initial TEE-based device size estimation was accurate with successful implantation at the first attempt. One patient required a change in initial device size and, therefore, required a second attempt for successful implantation. There was no peri-device leak immediately post-implantation in any patient; only one patient had a significant device leak on day 45 TEE requiring continuation of anticoagulation for four months until a successful device seal. There were no immediate or late complications up to 45 -days post-implantation.

\section{Conclusion}

Our experience shows no significant compromise in the efficiency and safety of the WATCHMAN implantation without contrast in patients with advanced CKD or a contrast allergy.

Categories: Cardiology, Nephrology

Keywords: watchman, left atrial appendage occlusion without contrast use, transesophageal echocardiography

\section{Introduction}

Since the Prospective Randomized Evaluation of the Watchman LAA Closure Device In Patients With Atrial Fibrillation Versus Long Term Warfarin Therapy (PREVAIL) study demonstrated its noninferiority to warfarin for thromboembolism prevention in non-valvular atrial fibrillation, left atrial appendage (LAA) occlusion is increasingly offered to patients who are unable to tolerate long-term anticoagulation [1]. Intraprocedural 


\section{Cureus}

real-time transesophageal echocardiographic (TEE) and fluoroscopic imaging are used to visualize anatomic structures, catheters/guidewires, and LAA occlusion devices [2]. Fluoroscopic guidance, in addition to radiation exposure, involves the use of nephrotoxic contrast. Over one-third of patients with an implanted LAA occlusion device have chronic kidney disease [3]. We report our experience performing LAA occlusion with the WATCHMAN (Boston Scientific, St. Paul, Minnesota) device, guided by TEE and fluoroscopy without contrast administration.

\section{Materials And Methods}

Consecutive procedure notes for all WATCHMAN devices implanted between June 2016 and June 2019 were screened to identify patients who did not receive contrast. The electronic records of these patients were reviewed for demographic information; comorbid conditions; baseline renal and liver function; 'Congestive heart failure, Hypertension, Age [>75 years], Stroke, Diabetes mellitus, Vascular disease, Age [65 to 74 years], Sex category' (CHA2DS2VASc) and 'Hypertension, Abnormal renal/liver function, Stroke, Bleeding history, Labile international normalized ratio, Elderly, Drugs/alcohol' (HAS-BLED) scores; reason(s) precluding safe use of contrast; and additional risk factors for bleeding or thrombosis not captured by the CHA2DS2VASC/HAS-BLED scores. Patients lacking complete information to calculate the CHA2DS2VASc/HAS-BLED score were excluded.

The following endpoints were assessed:

a. Efficiency

i) accuracy of device size estimation based on TEE-measured LAA dimensions was determined by the need to change the size of the device initially selected

ii) number of implantation attempts, irrespective of change in device size

iii) whether more than one device was used secondary to inaccurate initial size estimation or other procedural complexities

iv) successful LAA seal on TEE immediately and 45 -days post-implantation (peri-device leak of $<5 \mathrm{~mm}$ by color Doppler)

b. Safety-procedure-related complications, immediate and delayed (0-45 days)

Microsoft Excel (Microsoft Corporation, Redmond, Washington) was used for statistical analysis. Continuous variables are expressed as mean with standard deviation (mean +/- SD); categorical variables as percentages.

\section{Results}

The WATCHMAN device was implanted in 13 patients under TEE and fluoroscopic guidance only without any contrast use (Figures $1 A-1 D)$.

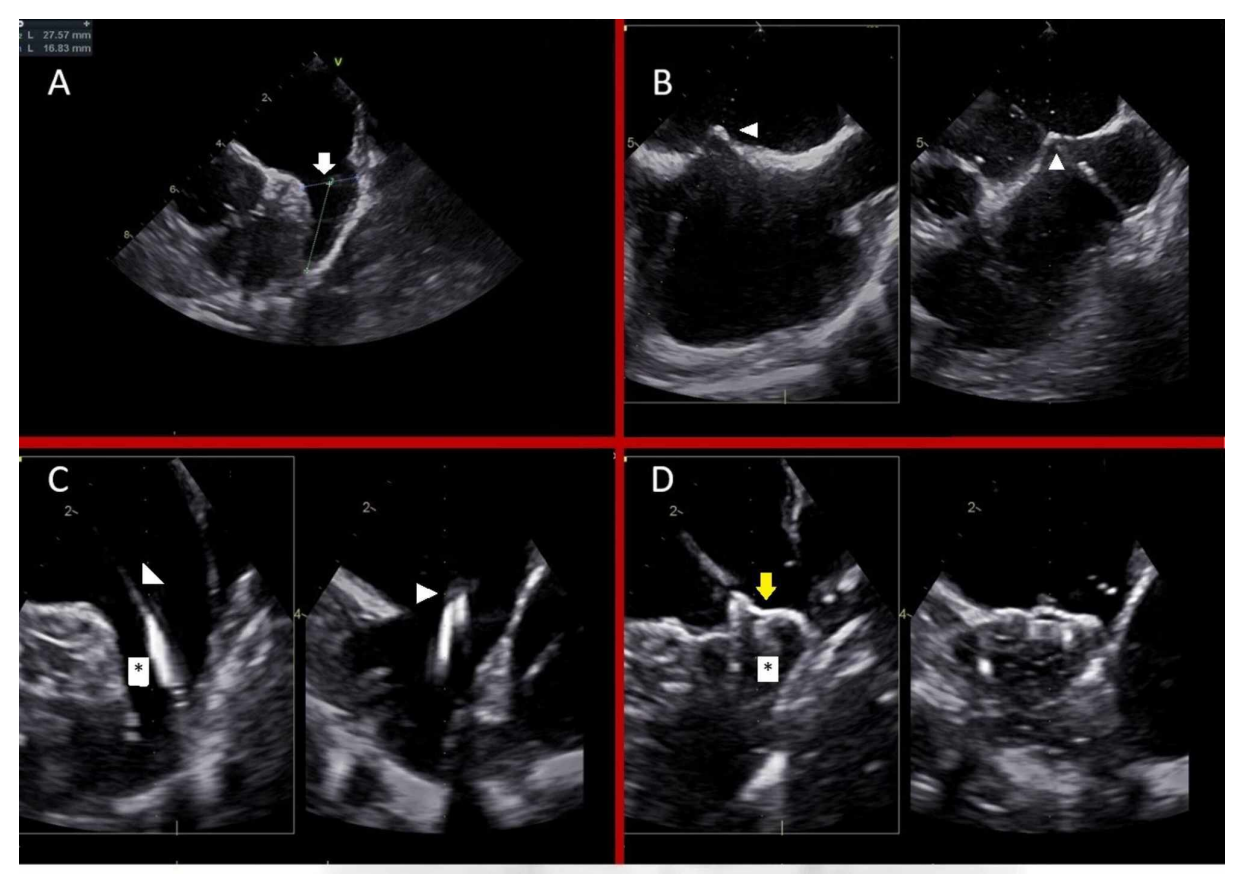

\section{FIGURE 1: TEE-guided WATCHMAN implantation}

Panel A: LAA measurement on appendage view (arrow). Panel B: Bicaval view showing tenting of the interatrial septum (arrowheads). Panel C: Appendage view: Delivery catheter (arrowheads) in LAA (asterisk). 


\section{Cureus}

Panel D: WATCHMAN (yellow arrow) implanted in LAA (asterisk).

TEE: Transesophageal echocardiography; LAA: Left atrial appendage

WATCHMAN: Boston Scientific, St. Paul, Minnesota

One patient was excluded because of incomplete information to calculate the CHA2DS2VASC/HAS-BLED scores. The final study cohort consisted of 12 patients with a mean age of 79.2 years and male predominance (Table 1).

\section{Baseline characteristics}

Mean age (years +/- SD)

Female

Congestive Heart Failure

Hypertension

Diabetes Mellitus

Stroke/TIA/Venous Thromboembolism

Vascular Disease $^{a}$

Abnormal Liver Function ${ }^{b}$

Abnormal Renal Function ${ }^{c}$

Mean eGFR $\left[\left(\mathrm{ml} / \mathrm{min} / 1.73 \mathrm{~m}^{2}\right)+/-\mathrm{SD}\right]$

Mean CHA2DS2VASc score +/- SD

Mean HAS-BLED score +/- SD $\mathrm{n}=12(\%)$

$79.2+/-5.44$

4 (33.3)

7 (58.3)

11 (91.7)

5 (41.7)

7 (58.3)

5 (41.7)

0 (0)

3 (75.0)

$46+/-17.4$

$5.50+/-1.24$

$4.08+/-1.08$

\section{TABLE 1: Baseline characteristics}

a Myocardial infarction, peripheral vascular disease, aortic plaque; b Total bilirubin $>$ twice upper-limit of normal or liver function tests $>3$ times upper limit of normal; c Serum $\mathrm{Cr}>2.2 \mathrm{mg} / \mathrm{dl}$, renal transplant or end-stage renal disease on hemodialysis

TIA: transient ischemic attack; eGFR: estimated glomerular filtration rate; CHA2DS2VASc: Congestive heart failure, Hypertension, Age [>75 years], Stroke, Diabetes mellitus, Vascular disease, Age [65 to 74 years], Sex category; HAS-BLED: Hypertension, Abnormal renal/liver function, Stroke, Bleeding history, Labile international normalized ratio, Elderly, Drugs/alcohol

The mean CHA2DS2VASc and HAS-BLED scores were $5.50(+/-1.24)$ and $4.08(+/-1.08)$, respectively. Reasons for LAA occlusion with WATCHMAN included non-variceal gastrointestinal bleeding requiring blood transfusions $(n=5)$, recurrent gastroesophageal variceal bleeding $(n=2)$, and worsening hematuria in the setting of urothelial cancer $(\mathrm{n}=1)$. Four patients received WATCHMAN to prevent bleeding associated with high fall risk: Elderly on triple anti-thrombotic therapy $(n=2)$, uncontrolled seizures $(n=1)$, and advanced Parkinson's disease $(\mathrm{n}=1)$.

Contrast use was avoided because of chronic kidney disease stage IV ( $n=5)$, rapidly progressive chronic kidney disease stage III $(\mathrm{n}=1)$, and contrast allergy $(\mathrm{n}=6)$.

Mean LAA depth and ostial diameter were $27.9+/-5.70 \mathrm{~mm}$ and $20.5+/-5.03 \mathrm{~mm}$, respectively. THE mean WATCHMAN device size was $28.0+/-4.11 \mathrm{~mm}$. In 11 out of 12 patients, initial TEE-based device size estimation was accurate with successful implantation on the first attempt (Table 2). 


\section{Cureus}

\section{Procedural Efficiency}

WATCHMAN implantation successful on initial attempt

\section{TABLE 2: Procedural efficiency}

a $<5 \mathrm{~mm}$ leak on Doppler ultrasound

WATCHMAN: Boston Scientific, St. Paul, Minnesota

One patient required a change in initial device size and, therefore, required a second attempt for successful implantation. There was no peri-device leak immediately post-implantation in any patient; only one patient had significant device leak on day 45 TEE, requiring continuation of anticoagulation for four months until successful device seal on repeat TEE. There were no immediate or late complications up to 45 days postimplantation (Table 3).

\begin{tabular}{|l|l|}
\hline Procedural Complications & $\mathrm{n=12}(\%)$ \\
\hline Cardiac tamponade & $0(0)$ \\
\hline Pericardial effusion/pericarditis & $0(0)$ \\
\hline Device embolization/stroke/death & $0(0)$ \\
Severe bleeding on post-WATCHMAN anticoagulation ${ }^{a}$ & $0(0)$ \\
\hline Non-severe bleeding on post- Watchman anticoagulation & $1(8.33)$
\end{tabular}

\section{TABLE 3: Procedural complications}

a Severe bleeding: hemodynamic instability, end-organ dysfunction, or requiring transfusion

WATCHMAN: Boston Scientific, St. Paul, Minnesota

\section{Discussion}

In 11 out of 12 patients, TEE without contrast fluoroscopy accurately measured ostial diameters and the depth of the LAA. This translated into appropriate WATCHMAN size estimation and successful implantation on the initial attempt. Only one patient required a change of device due to incorrect initial WATCHMAN size estimation (correctly re-measured with echocardiography). There were no periprocedural complications up to 45 days post WATCHMAN.

There are limited data on LAA occlusion guided solely by TEE. A single-center prospective study with 14 patients demonstrated successful and safe implantation of LAmbre (LAA occlusion device; Lifetech Scientific (Shenzhen) Co. Ltd., Shenzhen, China) with TEE only [4]. There is a single case report of TEEguided WATCHMAN implantation without contrast in an elderly patient with advanced chronic kidney disease, similar to the patients reported in our study [5]. About $40 \%$ of patients with an LAA occlusion device are estimated to have chronic kidney disease [3]. Patients with chronic kidney disease are at increased risk of both bleeding and thromboembolism. Evidence for direct-acting oral anticoagulants (DOACs) in chronic kidney disease is derived, at best, from post-hoc analyses. LAA occlusion has shown comparable efficacy in stroke prevention in patients with or without chronic kidney disease [6-8]. Avoiding contrast in LAA occlusion procedures offers the additional benefit of reducing the risk of contrast-associated acute kidney injury [9]. Notwithstanding its dependence on the acquisition of high-quality 3-D TEE images, our study suggests that contrast during WATCHMAN implantation may be avoided without compromising the efficiency or safety of the procedure. 
The limitations of our study are acknowledged. Although it is, to our knowledge, the largest study to-date on WATCHMAN implantation without contrast, the sample size is small and limited to a single center. Due to the retrospective nature of the study, it is unclear how the technique affects radiation exposure and procedure time. A direct comparison of WATCHMAN implantation with and without contrast is needed.

\section{Conclusions}

This is the largest reported, single-center study on successful LAA occlusion with the WATCHMAN device under TEE and fluoroscopy guidance without the use of contrast. Avoiding contrast, in our experience, does not significantly compromise the efficiency and safety of the procedure in patients with advanced CKD or contrast allergy.

\section{Additional Information \\ Disclosures}

Human subjects: Consent was obtained by all participants in this study. Animal subjects: All authors have confirmed that this study did not involve animal subjects or tissue. Conflicts of interest: In compliance with the ICMJE uniform disclosure form, all authors declare the following: Payment/services info: All authors have declared that no financial support was received from any organization for the submitted work. Financial relationships: All authors have declared that they have no financial relationships at present or within the previous three years with any organizations that might have an interest in the submitted work. Other relationships: All authors have declared that there are no other relationships or activities that could appear to have influenced the submitted work.

\section{References}

1. Holmes DR, Kar S, Price MJ, et al.: Prospective randomized evaluation of the watchman left atrial appendage closure device in patients with atrial fibrillation versus long-term warfarin therapy: the PREVAIL trial. J Am Coll Cardiol. 2014, 64:1-12. 10.1016/j.jacc.2014.04.029

2. Jungen C, Zeus T, Balzer J, et al.: Left atrial appendage closure guided by integrated echocardiography and fluoroscopy imaging reduces radiation exposure. PloS One. 2015, 2015:0140386. 10.1371/journal.pone.0140386

3. Kefer J, Tzikas A, Freixa X, et al.: Impact of chronic kidney disease on left atrial appendage occlusion for stroke prevention in patients with atrial fibrillation. Int J Cardiol. 2016, 207:335-340. 10.1016/j.ijcard.2016.01.003

4. Zhao J, Li F, Zhang Y, et al.: Left atrial appendage occlusion guided only by transesophageal echocardiography. Cardiol Res Pract. 2019, 2019:1376515. 10.1155/2019/1376515

5. Aminian A, Lempereur M, Ben Yedder M, Dubois P: Real-time fusion of echocardiography and fluoroscopy allowing successful implantation of a WATCHMAN device without contrast injection. Eurointervention. 2018, 14:174-175. 10.4244/EIJ-D-17-00398

6. Amabile N, Elhadad S, Roig C, Sebag F, Charles P, Caussin C: Left atrial appendage occlusion in elderly [Article in French]. Ann Cardiol Angeiol (Paris). 2018, 67:444-449. 10.1016/j.ancard.2018.10.001

7. Ronco F, Mazzone P, Hosseinian L, Genovesi S: Recent advances in stroke prevention in patients with atrial fibrillation and end-stage renal disease. Cardiorenal Med. 2017, 7:207-217. 10.1159/000470856

8. Manes M, Radin E, Pellù V, et al.: Monocentric experience of left atrial appendage occlusion among patients with advanced chronic kidney disease and non-valvular atrial fibrillation [Article in Italian]. G Ital Nefrol. 2020, 1:37.

9. Brockmeyer M, Wolff G, Krieger T, et al.: Kidney function stratified outcomes of percutaneous left atrial appendage occlusion in patients with atrial fibrillation and high bleeding risk. Acta Cardiol. 2019, 1:9. 10.1080/00015385.2019.1585643 